\title{
ON UNIFIED CLASS OF $\gamma$-SPIRALLIKE FUNCTIONS OF COMPLEX ORDER
}

\author{
T. M. SEOUDY
}

\begin{abstract}
In this paper, we obtain a necessary and sufficient condition for functions to be in an unified class of $\gamma$-spirallike functions of complex order. Some of our results generalize previously known results.
\end{abstract}

\section{INTRODUCTION}

Let $\mathcal{A}$ denote the class of functions of the form

$$
f(z)=z+\sum_{n=2}^{\infty} a_{n} z^{n},
$$

which are analytic in the open unit disk $\mathbb{U}=\{z \in \mathbb{C}:|z|<1\}$. Suppose that $f$ and $g$ are analytic in $\mathbb{U}$. We say that the function $f$ is subordinate to $g$ in $\mathbb{U}$, or $g$ superordinate to $f$ in $\mathbb{U}$, and we write $f \prec g$ or $f(z) \prec g(z)(z \in \mathbb{U})$, if there exists an analytic function $\omega$ in $\mathbb{U}$ with $\omega(0)=0$ and $|\omega(z)|<1$, such that $f(z)=g(\omega(z))(z \in \mathbb{U})$. If $g$ is univalent in $\mathbb{U}$, then the following equivalence relationship holds true (see [5] and [6]):

$$
f(z) \prec g(z) \Longleftrightarrow f(0)=g(0) \quad \text { and } \quad f(\mathbb{U}) \subset g(\mathbb{U}) \text {. }
$$

Let $\mathcal{S}$ be the subclass of $\mathcal{A}$ consisting of univalent functions. Let $\phi(z)$ be an analytic function with positive real part on $\phi$ with $\phi(0)=1, \phi^{\prime}(0)>0$ which maps the unit disk $\mathbb{U}$ onto a region starlike with respect to 1 which is symmetric with respect to the real axis. Let $\mathcal{S}^{*}(\phi)$ be the class of functions in $f \in \mathcal{S}$ for which

$$
\frac{z f^{\prime}(z)}{f(z)} \prec \phi(z),
$$

and $\mathcal{C}(\phi)$ class of functions in $f \in \mathcal{S}$ for which

$$
1+\frac{z f^{\prime \prime}(z)}{f^{\prime}(z)} \prec \phi(z) \text {. }
$$

2010 Mathematics Subject Classification. Primary: 30C45. Secondary: 30D30, 33D20.

Key words and phrases. Analytic function, univalent, starlike, convex, subordination. 
These classes were introduced and studied by Ma and Minda [4]. Ravichandran et al. [10] defined classes $\mathcal{S}_{b}^{*}(\phi)$ and $\mathcal{C}_{b}(\phi)$ of complex order defined as follows :

$$
\mathcal{S}^{*}(\phi ; b)=\left\{f \in \mathcal{A}: 1+\frac{1}{b}\left(\frac{z f^{\prime}(z)}{f(z)}-1\right) \prec \phi(z) \quad\left(b \in \mathbb{C}^{*}=\mathbb{C} \backslash\{0\}\right)\right\}
$$

and

$$
\mathcal{C}(\phi ; b)=\left\{f \in \mathcal{A}: 1+\frac{1}{b} \frac{z f^{\prime \prime}(z)}{f^{\prime}(z)} \prec \phi(z) \quad\left(b \in \mathbb{C}^{*}\right)\right\} .
$$

From (1.4) and (1.5), we have

$$
f \in \mathcal{C}(\phi ; b) \Longleftrightarrow z f^{\prime} \in \mathcal{S}^{*}(\phi ; b) .
$$

Now, we introduce a more general class of $\gamma$-spirallike functions of complex order $\mathcal{T}(\phi ; \lambda, b)$ as follow:

Definition 1. Let $\phi(z)$ be an analytic function with positive real part on $\phi$ with $\phi(0)=1, \phi^{\prime}(0)>0$ which maps the unit disk $\mathbb{U}$ onto a region starlike with respect to 1 which is symmetric with respect to the real axis. Then the class $\mathcal{T}^{\gamma}(\phi ; \lambda, b)$ consists of all analytic functions $f \in \mathcal{A}$ satisfying:

$$
\begin{gathered}
1+\frac{e^{i \gamma}}{b \cos \gamma}\left[(1-\lambda) \frac{z f^{\prime}(z)}{f(z)}+\lambda\left(1+\frac{z f^{\prime \prime}(z)}{f^{\prime}(z)}\right)-1\right] \prec \phi(z) \\
\left(|\gamma|<\frac{\pi}{2}, b \in \mathbb{C}^{*} ; \lambda \geq 0\right) .
\end{gathered}
$$

We note that

(i) $\mathcal{T}^{\gamma}\left(\frac{1+z}{1-z} ; 0, b\right)=\mathcal{S}^{\gamma}(b)$ and $\mathcal{T}^{\gamma}\left(\frac{1+z}{1-z} ; 1, b\right)=\mathcal{C}^{\gamma}(b)\left(|\gamma|<\frac{\pi}{2}, b \in \mathbb{C}^{*}\right)$ ( Al-Oboudi and Haidan [1] and Aouf et al. [2] );

(ii) $\mathcal{T}^{0}(\phi ; 0, b)=\mathcal{S}^{*}(\phi ; b)$ and $\mathcal{T}^{0}(\phi ; 1, b)=\mathcal{C}(\phi ; b)$ ( Ravichandran et al. $[10])$

(iii) $\mathcal{T}^{0}(\phi ; 0,1)=\mathcal{S}^{*}(\phi)$ and $\mathcal{T}^{0}(\phi ; 1,1)=\mathcal{C}(\phi)$ ( Ma and Minda [4]);

(iv) $\mathcal{T}^{0}\left(\frac{1+(1-2 \alpha) z}{1-z} ; 0, b\right)=\mathcal{S}_{\alpha}^{*}(b)$ and $\mathcal{T}^{0}\left(\frac{1+(1-2 \alpha) z}{1-z} ; 1, b\right)=\mathcal{C}_{\alpha}(b)(0 \leq \alpha$ $\left.<1 ; b \in \mathbb{C}^{*}\right)$ ( Frasin [3] );

(v) $\mathcal{T}^{0}\left(\frac{1+z}{1-z} ; 0, b\right)=\mathcal{T}^{0}\left(\frac{1+(2 b-1) z}{1-z} ; 0,1\right)=\mathcal{S}^{*}(b)\left(b \in \mathbb{C}^{*}\right)$ (Nasr and Aouf [8] and Wiatrowski [15] );

(vi) $\mathcal{T}^{0}\left(\frac{1+z}{1-z} ; 1, b\right)=\mathcal{T}^{0}\left(\frac{1+(2 b-1) z}{1-z} ; 1,1\right)=\mathcal{C}(b)\left(b \in \mathbb{C}^{*}\right)$ (Nasr and Aouf [7] and Wiatrowski [15] );

(vii) $\mathcal{T}^{0}\left(\frac{1+z}{1-z} ; 0,1-\alpha\right)=\mathcal{T}^{0}\left(\frac{1+(1-2 \alpha) z}{1-z} ; 0,1\right)=\mathcal{S}^{*}(\alpha)$ and $\mathcal{T}^{0}\left(\frac{1+z}{1-z} ; 1\right.$, $1-\alpha)=\mathcal{T}^{0}\left(\frac{1+(1-2 \alpha) z}{1-z} ; 1,1\right)=\mathcal{C}(\alpha)(0 \leq \alpha<1)$ (Robertson [11]). 
Motivated essentially by the aforementioned works, we obtain certain necessary and sufficient conditions for the unified class of functions $\mathcal{T}^{\gamma}(\phi ; \lambda, b)$ which we have defined. The motivation of this paper is to generalize the results obtained by Ravichandran et al. [10], Aouf et al. [2], Srivastava and Lashin [14] and also Obradovic et al. [9].

\section{MAIN RESULTS}

Unless otherwise mentioned, we assume throughout the sequel that $|\gamma|<\frac{\pi}{2}, b \in$ $\mathbb{C}^{*}, \lambda \geq 0$ and all powers are understood as principle values. To prove our main result, we need the following lemmas.

Lemma 1 ([12]). Let $\phi$ be a convex function defined on $\mathbb{U}, \phi(0)=1$. Define $F(z)$ by

$$
F(z)=z \exp \left(\int_{0}^{z} \frac{\phi(t)-1}{t} d t\right) .
$$

Let $p(z)=1+p_{1} z+p_{2} z^{2}+\cdots$ be analytic in $\mathbb{U}$. Then

$$
1+\frac{z q^{\prime}(z)}{q(z)} \prec \phi(z)
$$

if and only if for all $|s| \leq 1$ and $|t| \leq 1$, we have

$$
\frac{p(t z)}{p(s z)} \prec \frac{s F(t z)}{t F(s z)} .
$$

Lemma 2 ([6]). Let $q(z)$ be univalent in $\mathbb{U}$ and let $\varphi(z)$ be analytic in a domain containing $q(\mathbb{U})$. If $\frac{z q^{\prime}(z)}{q(z)}$ is starlike, then

$$
z p^{\prime}(z) \varphi(p(z)) \prec z q^{\prime}(z) \varphi(q(z))
$$

then $p(z) \prec q(z)$ and $q(z)$ is the best dominant.

Theorem 1. Let $\phi(z)$ and $F(z)$ be as in Lemma 1. The function $f \in \mathcal{T}^{\gamma}(\phi ; \lambda, b)$ if and only if for all $|s| \leq 1$ and $|t| \leq 1$, we have

$$
\left[\left(\frac{s f(t z)}{t f(s z)}\right)^{1-\lambda}\left(\frac{f^{\prime}(t z)}{f^{\prime}(s z)}\right)^{\lambda}\right] \frac{e^{i \gamma}}{b \cos \gamma} \prec \frac{s F(t z)}{t F(s z)} .
$$

Proof. Define the function $p(z)$ by

$$
p(z)=\left[\frac{f(z)}{z}\left(\frac{z f^{\prime}(z)}{f(z)}\right)^{\lambda}\right]^{\frac{e^{i \gamma}}{b \cos \gamma}} \quad(z \in \mathbb{U}) .
$$


Taking logarithmic derivative of (2.5), we get

$$
1+\frac{z p^{\prime}(z)}{p(z)}=1+\frac{e^{i \gamma}}{b \cos \gamma}\left[(1-\lambda) \frac{z f^{\prime}(z)}{f(z)}+\lambda\left(1+\frac{z f^{\prime \prime}(z)}{f^{\prime}(z)}\right)-1\right] .
$$

Since $f \in \mathcal{T}(\phi ; \lambda, b)$, then we have

$$
1+\frac{z p^{\prime}(z)}{p(z)} \prec \phi(z)
$$

and the result now follows from Lemma 1.

Putting $\lambda=\gamma=0$ in Theorem 1, we obtain the following result of Shanmugam et al. [13].

Corollary 1. Let $\phi(z)$ and $F(z)$ be as in Lemma 1. The function $f \in \mathcal{S}^{*}(\phi ; b)$ if and only if for all $|s| \leq 1$ and $|t| \leq 1$, we have

$$
\left(\frac{s f(t z)}{t f(s z)}\right)^{\frac{1}{b}} \prec \frac{s F(t z)}{t F(s z)} .
$$

For $\lambda=1$ and $\gamma=0$ in Theorem 1, we obtain the following result of Shanmugam et al. [13].

Corollary 2. Let $\phi(z)$ and $F(z)$ be as in Lemma 1. The function $f \in \mathcal{C}(\phi ; b)$ if and only if for all $|s| \leq 1$ and $|t| \leq 1$, we have

$$
\left(\frac{f^{\prime}(t z)}{f^{\prime}(s z)}\right)^{\frac{1}{b}} \prec \frac{s F(t z)}{t F(s z)} .
$$

Theorem 2. Let $\phi(z)$ be starlike with respect to 1 and $F(z)$ given by (2.1) be starlike. If $f \in \mathcal{T}^{\gamma}(\phi ; \lambda, b)$, then we have

$$
\frac{f(z)}{z}\left(\frac{z f^{\prime}(z)}{f(z)}\right)^{\lambda} \prec\left(\frac{F(z)}{z}\right)^{\frac{b \cos \gamma}{e^{i \gamma}}} .
$$

Proof. Let $p(z)$ be given by (2.5) and $q(z)$ be given by

$$
q(z)=\frac{F(z)}{z} \quad(z \in \mathbb{U})
$$

After a simple computation we obtain

$$
1+\frac{z p^{\prime}(z)}{p(z)}=1+\frac{e^{i \gamma}}{b \cos \gamma}\left[(1-\lambda) \frac{z f^{\prime}(z)}{f(z)}+\lambda\left(1+\frac{z f^{\prime \prime}(z)}{z f^{\prime}(z)}\right)-1\right]
$$

and

$$
\frac{z q^{\prime}(z)}{q(z)}=\frac{z F^{\prime}(z)}{F(z)}-1=\phi(z)-1
$$


Since $f \in \mathcal{T}^{\gamma}(\phi ; \lambda, b)$, we have

$$
\frac{z p^{\prime}(z)}{p(z)} \prec \frac{z q^{\prime}(z)}{q(z)} .
$$

The result now follows by an application of Lemma 2 .

For $\phi(z)=\frac{1+z}{1-z}$ and $\lambda=0$ in Theorem 2, we get the following result of Aouf et al. [2].

Corollary 3. If $f \in \mathcal{S}^{\gamma}(b)$, then we have

$$
\frac{f(z)}{z} \prec(1-z)^{-2 b e^{-i \gamma} \cos \gamma} .
$$

For $\phi(z)=\frac{1+z}{1-z}$ and $\lambda=1$ in Theorem 2, we get the following result of Aouf et al. [2].

Corollary 4. If $f \in \mathcal{C}^{\gamma}(b)$, then we have

$$
f^{\prime}(z) \prec(1-z)^{-2 b e^{-i \gamma} \cos \gamma} .
$$

Putting $\lambda=\gamma=0$ in Theorem 2, we obtain the following results of Shanmugam et al. [13].

Corollary 5. Let $\phi(z)$ be starlike with respect to 1 and $F(z)$ given by (2.1) be starlike. If $f \in \mathcal{S}^{*}(\phi ; b)$, then we have

$$
\frac{f(z)}{z} \prec\left(\frac{F(z)}{z}\right)^{\frac{b \cos \gamma}{e^{i \gamma}}} .
$$

Taking $\phi(z)=\frac{1+A z}{1+B z}(-1 \leq B<A \leq 1)$ in Theorem 2, we get the following corollary:

Corollary 6. If $f \in \mathcal{T}^{\gamma}\left(\frac{1+A z}{1+B z} ; \lambda, b\right)(-1 \leq B<A \leq 1)$, then we have

$$
\frac{f(z)}{z}\left(\frac{z f^{\prime}(z)}{f(z)}\right)^{\lambda} \prec(1+B z)^{\frac{(A-B) b \cos \gamma}{B e^{i \gamma}}}(B \neq 0) .
$$

For $\phi(z)=\frac{1+z}{1-z}$ and $\lambda=\gamma=0$ in Theorem 2, we get the following result of Obradovic et al. [9], and Srivastava and Lashin [14].

Corollary 7. If $f \in \mathcal{S}^{*}(b)$, then we have

$$
\frac{f(z)}{z} \prec(1-z)^{-2 b} .
$$


For $\phi(z)=\frac{1+z}{1-z}, \gamma=0$ and $\lambda=1$ in Theorem 2, we get the following result of Obradovic et al. [9], and Srivastava and Lashin [14].

Corollary 8. If $f \in \mathcal{C}(b)$, then we have

$$
f^{\prime}(z) \prec(1-z)^{-2 b} .
$$

\section{REFERENCES}

[1] F. M. Al-Oboudi and M. M. Haidan, Spirallike functions of complex order, J. Nat. Geom., 19 (2000), 53-72.

[2] M. K. Aouf, F. M. Al-Oboudi and M. M. Haidan, On some results for $\lambda$-spirallike and $\lambda$ Robertson functions of complex order, Publ. Instit. Math. Belgrade, 77 (91) (2005), 93-98.

[3] B. A. Frasin, Family of analytic functions of complex order, Acta Math. Acad. Paedagog. Nyházi. (N. S.), 22 (2) (2006), 179-191.

[4] W. C. Ma and D. Minda, A unified treatment of some special classes of univalent functions, in Proceedings of the Conference on Complex Analysis (Tianjin, 1992), 157-169, Internat. Press, Cambridge, MA.

[5] S. S. Miller and P. T. Mocanu, Differential subordinations and univalent functions, Michigan Math. J., 28 (1981), 157-171.

[6] S. S. Miller and P. T. Mocanu, Differential Subordinations: Theory and Applications, Serieson Monographs and Textbooks in Pure and Applied Mathematics, Vol. 225, Marcel Dekker, New York and Basel, 2000.

[7] M. A. Nasr and M. K. Aouf, On convex functions of complex order, Mansoura Bull. Sci., 8 (1982), 565-582.

[8] M. A. Nasr and M. K. Aouf, Starlike function of complex order, J. Nat. Sci. Math., 25 (1985), $1-12$

[9] M. Obradovic, M. K. Aouf and S. Owa, On some results for starlike functions of complex order, Publ. Inst. Math., Nouv. Ser. 46 (60) (1989), 79-85.

[10] V. Ravichandran, Y. Polatoglu, M. Bolcal and A. Sen, Certain subclasses of starlike and convex functions of complex order, Hacettepe J. Math. Stat., 34 (2005), 9-15.

[11] M. S. Robertson, On the theory of univalent functions, Ann. Math., 37 (1936), 374-408.

[12] St. Ruscheweyh, Convolutions in Geometric Function Theory, Presses Univ. Montreal, Montreal, Que., 1982.

[13] T. N. Shanmugam, S. Sivasubramanian and S. Kavitha, On certain subclasses of functions of complex order, South. Asian Bull. Math., 33 (2009), 535-541.

[14] H. M. Srivastava and A.Y. Lashin, Some applications of the Briot-Bouquetdifferential subordination, J. Inequal. Pure Appl. Math., 6 (2) (2005), Art. 41, 1-7.

[15] P. Wiatrowski, On the coefficients of some family of holomorphic functions, Zeszyty Nauk. Uniw. Łódz Nauk. Mat.-Przyrod., 39 (1970), 75-85.

(Received: May 12, 2013)

(Revised: December 13, 2013)
Department of Mathematics Faculty of Science, Fayoum University Fayoum 63514, Egypt tmseoudy@gmail.com 